\title{
Dicer Enhances Bevacizumab-Related Inhibition of Hepatocellular Carcinoma via Blocking the Vascular Endothelial Growth Factor Pathway
}

\author{
Cuiju Wang' \\ Yalei Lv ${ }^{2}$ \\ Ziyue Sha $\mathbb{1}^{3}$ \\ Jingjing Zhang ${ }^{3}$ \\ Jianhua $\mathrm{Wu}^{4}$ \\ Yixin $\mathrm{Qi}^{5}$ \\ Zhanjun $\mathrm{Guo}^{3}$ \\ 'Department of Gynaecology \\ Ultrasound, The Fourth Hospital of \\ Hebei Medical University, Shijiazhuang, \\ People's Republic of China; ${ }^{2}$ Department \\ of Medical Oncology, The Fourth \\ Hospital of Hebei Medical University, \\ Shijiazhuang, People's Republic of China; \\ ${ }^{3}$ Department of Immunology and \\ Rheumatology, The Fourth Hospital of \\ Hebei Medical University, Shijiazhuang, \\ People's Republic of China; ${ }^{4}$ Animal \\ Center, The Fourth Hospital of Hebei \\ Medical University, Shijiazhuang, People's \\ Republic of China; ${ }^{5}$ Breast Center, The \\ Fourth Hospital of Hebei Medical \\ University, Shijiazhuang, People's Republic \\ of China
}

Purpose: Vascular endothelial growth factor (VEGF) family members contribute greatly to the development and angiogenesis of hypervascular hepatocellular carcinoma (HCC). We have previously shown that Dicer inhibited HCC growth. In this study, we aimed to determine the relationship between Dicer and VEGF in HCC.

Methods: Gain-of-function studies were performed to determine the effect of different treatments on the proliferation, migration, and invasion of HCC cells. Expression of VEGF-A in xenograft tumor tissues was analysed using Western blotting, and that of CD31 using immunohistochemical analysis.

Results: We found that Dicer inhibited proliferation, migration and invasion of HCC cells by suppressing VEGF-A expression. Interestingly, VEGF-A165, which is the most prominent VEGF-A isoform, counteracted Dicer-induced inhibition of HCC cells. In addition, a monoclonal anti-VEGF antibody (bevacizumab) enhanced Dicer-induced inhibition of HCC in vitro and in vivo. Further, immunohistochemical analysis of CD31 indicated bevacizumab and Dicer synergized to reduce tumor microvessel density.

Conclusion: Our data demonstrated that Dicer enhanced bevacizumab-related inhibition of HCC cell via the VEGF pathway; therefore, Dicer in coordination with bevacizumab may provide another potential approach for HCC therapy.

Keywords: hepatocellular carcinoma, vascular endothelial growth factor, Dicer, bevacizumab, microvessel density

\section{Introduction}

Primary liver cancer (PLC) is the sixth most common diagnosed cancer worldwide, with approximately 906,000 new cases and 830,000 deaths, rendering it the third leading cause of cancer mortality in $2020 .{ }^{1}$ In approximately $50 \%$ new PLC cases that occur in China, chronic hepatitis B virus (HBV) infection is the main risk factor. ${ }^{2,3}$ Hepatocellular carcinoma (HCC) is the most common liver cancer and accounts for over $75-85 \%$ of PLC. Potentially curative surgical resection, local ablation therapy, and radiation intervention are options for patients with early-stage HCC. However, most patients cannot undergo such treatments due to intrahepatic or distant metastasis. ${ }^{4}$

As angiogenesis is mediated by the vascular endothelial growth factor (VEGF) and VEGF receptor (VEGFR) contributes to the invasion and metastasis of hypervascular $\mathrm{HCC}$, antitumor angiogenesis is a potential target for HCC treatment. ${ }^{5}$ Although many antitumor angiogenesis agents, including antibodies and tyrosine kinase inhibitors (TKIs) such as brivanib, sunitinib, linifanib, everolimus and axitinib have failed in
Correspondence: Yixin Qi; Zhanjun Guo Email 13932153600@I39.com; zjguo5886@aliyun.com 
HCC therapy, ${ }^{6-11}$ sorafenib and lenvatinib have shown to improve outcomes of HCC patients and recommended by National Comprehensive Cancer Network (NCCN) as the first-line treatment for HCC. ${ }^{12,13}$ Moreover, apatinib and ramucirumab have succeeded in the therapy of HCC patients those who have failed first-line treatment with sorafenib. ${ }^{14,15}$ These data demonstrate that anti-VEGF/VEGFR therapy is an effective treatment for HCC. Bevacizumab, which is a monoclonal antibody against VEGF, is used for the treatment of advanced colorectal, lung, breast, and brain cancers. ${ }^{16}$ Currently, bevacizumab has been used in few clinical trials in $\mathrm{HCC}$ as single agent. A phase II trial showed that the median progression-free survival (mPFS) was 6.9 months in 46 HCC patients who underwent bevacizumab therapy, ${ }^{17}$ suggesting that bevacizumab is potentially effective treatment for advanced HCC. As antitumor angiogenesis agents can normalize tumor vessels and change the immunosuppressive environment of HCC, combination therapy with immune checkpoint inhibitors and antitumor angiogenesis agents demonstrated a synergistic antitumor effect. ${ }^{18}$ The combination therapy of programmed cell death 1 ligand 1 (PD-L1) inhibitor atezolizumab with bevacizumab improved mPFS significantly, when compared with sorafenib (6.8 versus 4.3 months, HR 0.59 ; 95\% CI: $0.47-0.76$; $p<0.0001) .{ }^{19}$ However, atezolizumab is too expensive for the developing countries, thus bevacizumab may be HCC treatment regime.

MicroRNAs (miRNAs) are 18-25 nt short noncoding RNA sequences that bind to the 3 '-untranslated region (3'UTR) of target mRNAs to modulate gene expression programs by regulating their translation and stability. ${ }^{20-22}$ Dicer is a cytoplasmic RNaseIII enzyme that cleaves premicroRNAs into mature microRNAs and short interfering RNAs in the cytoplasm. ${ }^{23}$ Several lines of evidence have demonstrated that Dicer downregulation was associated with poor prognosis in some human cancers, such as HCC, renal cell carcinoma (RCC), gastric cancer, breast cancer, colorectal cancer and chronic lymphocytic leukemia, thus acting as a tumor suppressor. ${ }^{24-29}$ As Dicer suppressed VEGF-A, a key prototypical member of the VEGF family in $\mathrm{RCC}^{30}$ we investigated whether Dicer may control HCC progression through the VEGF pathway.

\section{Materials and Methods}

\section{Cell Culture and Transduction}

The human $\mathrm{HCC}$ cell line $\mathrm{HuH}-7$ was purchased from Procell Life Science \& Technology Co., Ltd (Wuhan, Hubei, China), and SMMC-7721 was acquired from the
Cell Bank of the Chinese Academy of Sciences (Shanghai, China). Both cell lines were characterized by mycoplasma detection (Supplementary Figure S1), DNA fingerprinting, isozyme detection and cell viability. HCC cells were cultured in DMEM high-glucose medium (GibcoTMLife Technologies, NY, USA) with $10 \%$ fetal bovine serum (FBS) (GibcoTMLife Technologies) in a humidified incubator containing a $5 \% \mathrm{CO}_{2}$ atmosphere at $37{ }^{\circ} \mathrm{C}$.

To overexpress Dicer, approximately $1 \times 10^{5} \mathrm{HCC}$ cells were incubated with a Dicer-overexpressing lentivirus (pCMV-Dicer) (GeneCopoeia, Rockville, MD, USA) tagged with green fluorescent protein (GFP) as described previously. $^{31}$ As a negative control, HCC cells were infected with control lentivirus (pCMV-NC) (GeneCopoeia, Rockville, MD, USA). GFP expression was observed under the microscope $72 \mathrm{~h}$ after infection to determine transduction efficiency. Seventy-two hours post-transduction, cells were selected with puromycin (2 $\mu \mathrm{g} / \mathrm{mL}$ ) for 2 weeks to generate stable cell lines. Successful overexpression of Dicer was confirmed with Western blotting using an anti-Dicer antibody (Abcam, Cambridge, UK).

\section{Western Blot}

Western blotting was performed, as previously described, ${ }^{32}$ to confirm Dicer overexpression and the level of VEGF-A. Equal protein quantities from total cell lysates were subjected to SDS-PAGE electrophoresis and transferred to PVDF membranes. Membranes were blocked for $2 \mathrm{~h}$ in blocking buffer ( $5 \%$ non-fat dry milk in Tris-buffered saline with $0.1 \%$ Tween 20 ) at room temperature and incubated overnight at $4^{\circ} \mathrm{C}$ with the following primary antibodies: anti-Dicer (dilution 1:1000; Abcam, Cambridge, UK), anti-VEGF-A (dilution 1:1000; Abcam, Cambridge, UK) and anti- $\beta$-actin (dilution 1:5000; Abcam, Cambridge, $\mathrm{UK}$ ), followed by incubation with an anti-mouse IgG antibody (Abcam, Cambridge, UK) at a dilution of 1:5000. The relative intensities of protein bands were visualized using ECL (BD, San Diego, CA).

\section{Cell Proliferation Assay}

VEGF-A165 cytokine was purchased from Meilun Biotechnology Co. Ltd. (Dalian, China), and bevacizumab was purchased from Roche pharma (South San Francisco, CA). We used Cell Counting Kit-8 (CCK-8; Dojindo, Kumamoto, Japan) to measure cell proliferation. ${ }^{33}$ According to the manufacturer's protocol, approximately $1 \times 10^{4}$ cells were seeded into 96 -well plates with $100 \mu \mathrm{L}$ 
medium per well. Cell proliferation was determined at different time points, including 0, 12, 24, 48, 72 and 96 $\mathrm{h}$ after a $2 \mathrm{~h}$ incubation with $10 \mu \mathrm{L}$ of CCK-8. Absorbance in each well was measured at $450 \mathrm{~nm}$ wavelength by a microplate reader (Bio-Rad, Hercules, CA).

\section{Wound Healing Assay}

A wound healing assay was performed to determine the migration ability of the cells. ${ }^{34}$ Briefly, cells were seeded on 6-well plates. After cells reached approximately $100 \%$ confluence, the surface of the plates was scratched linearly with a $200-\mu \mathrm{L}$ pipette tip. Cells were washed twice with PBS and cultured in DMEM medium with 2\% FBS. Images were captured using an inverted microscopy (Nikon, Tokyo, Japan) at 0 and $24 \mathrm{~h}$. Healing rates were calculated as the width of a wound at $24 \mathrm{~h}$ divided by the initial width. ${ }^{35}$

\section{Invasion Assay}

Cell invasion was determined using 24-well transwell chambers with $8-\mu \mathrm{m}$ pore size (Corning, New York, NY) precoated with $1 \mathrm{mg} / \mathrm{mL}$ BD Matrigel (BD Biosciences, NJ). ${ }^{36}$ Before the invasion assay, cells were cultured for $24 \mathrm{~h}$ in DMEM medium with $2 \%$ FBS. In the upper compartment of the chamber, approximately $1 \times 10^{5}$ cells were added to DMEM without FBS, while $500 \mu \mathrm{L}$ of DMEM medium with $10 \%$ FBS were added to the lower chamber. After being incubated at $37 \mathrm{C}$ in a $5 \% \mathrm{CO}_{2}$ atmosphere for $24 \mathrm{~h}$, cells invaded into the underside were then washed, fixed with $4 \%$ paraformaldehyde and stained with $0.5 \%$ crystal violet. Stained cells were counted with an inverted microscopy (Nikon, Tokyo, Japan) in five random fields for each membrane (magnification 200×).

\section{Mouse Xenograft Tumor Model}

This study was approved by the Ethics Board of the Animal Ethics Committee of the Fourth Hospital of Hebei Medical University. Sixteen 4-week-old athymic nude BALB/c mice were purchased from Charles River Laboratories [Beijing, China; permission no. SCXK (Jing) 2016-0006]. Nude mice were housed and treated in accordance with the guidelines established by the National Institutes of Health Guide for the Care and Use of Laboratory Animals. $^{37}$

Xenograft tumors were generated with subcutaneous injection of $1 \times 10^{7}$ SMMC-7721 treated with pCMVDicer or pCMV-NC in $0.2 \mathrm{~mL}$ medium into the shoulder of nude mice. ${ }^{38}$ Seven days after tumor cell injection, mice were divided into the following four groups (four mice per group): pCMV-NC (group 1), pCMV-NC plus bevacizumab (group 2), pCMV-Dicer (group 3), and pCMV-Dicer plus bevacizumab (group 4). Groups 2 and 4 were intraperitoneally injected with $20 \mathrm{mg} / \mathrm{kg}$ bevacizumab at a concentration of $2.5 \mathrm{mg} / \mathrm{mL}$ once every 3 days for 3 weeks, while groups 1 and 3 were intraperitoneally injected with $8 \mathrm{~mL} / \mathrm{kg}$ saline as the negative control. Tumor growth and weight were measured every 7 days, and tumor volume was calculated according to the following formula: Volume $=$ Length $\times(\text { Width })^{2} / 2{ }^{39}$ In vivo green fluorescent images were acquired with NightOwl Bioimager (Berthold Technologies, Bad Wildbad, Germany) at timepoint of 18 and 28 days after implantation. The fluorescent intensity was analyzed by WinLight32 software package (Berthold Technologies).

\section{VEGF-A Protein Expression in Xenograft Tumors}

For xenograft tumors, $20 \mathrm{mg}$ of tissue was added to 100 mL RIPA lysis buffer (Zomanbio, Beijing, China), homogenized, and centrifuged at 13,000 g for $15 \mathrm{~min}$. Protein concentration was determined by a BCA Protein Assay Kit (Zomanbio, Beijing, China) according to the manufacturer's protocol. Then, the protein level of VEGF-A was examined with Western blotting, as described previously. For quantification analysis, the total density of each band was analyzed using Image-J software (National Institutes of Health, Bethesda, USA).

\section{Immunohistochemical Analysis}

After mice were sacrificed, tumor samples from each group were harvested, fixed in 4\% formaldehyde for 24 $\mathrm{h}$, then embedded in paraffin. Five-micrometer sections were immunostained with CD31 (Abcam, Cambridge, UK). For semi-quantification of microvessel density (MVD), positive staining was defined with the Weidner method: a microvessel was counted when cells or cell clusters were stained brown with CD31 with a clear separation from the surrounding tissues. Areas of highest neovascularization were found by scanning the tumor sections at low magnification $(100 \times)$, and then individual microvessels were counted at high magnification $(200 \times) .{ }^{40}$

\section{miRNA Microarray Analysis}

RNA samples from pCMV-Dicer and pCMV-NC group cells (three samples for each group) were examined with 
microarray analysis at Cnkingbio Biotechnology Corporation (Beijing, China), using FlashTag Biotin HSR RNA Labeling Kit (Affymetrix, Santa Clara, CA). Labeling and hybridization were performed according to the manufacturer's instructions. Microarray for miRNAs was manufactured and processed as described. ${ }^{41}$ Microarrays were scanned on GeneChip Scanner 3000 (Affymetrix), and data were analyzed using the GeneChip Command Console software (Affymetrix).

\section{Statistical Analysis}

All statistical analyses were performed using the Statistical Package for the Social Sciences (SPSS Company, version 21.0; Chicago, IL, USA). Results were presented as the mean \pm standard deviation. Two experimental groups were performed using Student's $t$-test after tested for normality. Multiple groups were compared using a one-way analysis of variance (ANOVA) first. The least significant difference $t$-test was applied, if the overall difference was statistically significant. A $p$-value $\leq 0.05$ was considered statistically significant.

\section{Results}

\section{Dicer Downregulates VEGF-A Expression in $\mathrm{HCC}$}

Successful overexpression of Dicer was confirmed with Western Blotting (Figures 1A and 2A). We then assessed the relationship between Dicer and VEGF-A in the HCC cell lines SMMC-7721 and HuH-7. As shown in Figures $1 \mathrm{~A}$ and $2 \mathrm{~A}$, the level of VEGF-A was dramatically decreased upon Dicer overexpression. These data demonstrated that Dicer could downregulate VEGF-A expression in HCC.

\section{VEGF-AI65 Counteracts the Growth Inhibition Induced by Dicer in HCC Cells}

We examined the Dicer-induced effect on HCC cells by comparing HCC cells infected with pCMV-Dicer or pCMV-NC, and found that the proliferation of pCMVDicer cells significantly decreased 48 to 96 hours after infection (Figures 1B, C, 2D and E, $p<0.05$ ); moreover, the ability of $\mathrm{HCC}$ cells to migrate decreased dramatically in pCMV-Dicer cells (Figures 1D, E, 2F and G, $p<0.01$ ), together with their invasive capacity (Figures $1 \mathrm{~F}, \mathrm{G}, 2 \mathrm{H}$ and I, $p<0.01$ ). Based on these data, we examined whether Dicer inhibited HCC proliferation, migration, and invasion via the VEGF pathway.
As VEGF-A165 is the most prominent VEGF-A isoform involved in HCC angiogenesis, ${ }^{42,43}$ we added VEGFA165 to the medium of the pCMV-Dicer cell culture to investigate its effect on HCC cell growth. Proliferation of pCMV-Dicer cells significantly increased 48 to $96 \mathrm{~h}$ after addition of 50 and $100 \mathrm{ng} / \mathrm{mL}$ VEGF-A165 to the medium (Figure $2 \mathrm{~B}$ and $\mathrm{C}, p<0.05$ ), therefore, $50 \mathrm{ng} / \mathrm{mL}$ VEGFA165 was used for subsequent analysis.

VEGF-A165 promoted the proliferation from 48 to 96 $\mathrm{h}$ (Figure $2 \mathrm{D}$ and $\mathrm{E}, p<0.05$ ), migration (Figure $2 \mathrm{~F}$ and $\mathrm{G}$, $p=0.001$ ) and invasion (Figure $2 \mathrm{H}$ and $\mathrm{I}, p=0.01$ ) in pCMV-NC infected SMMC-7721 cells. In addition, VEGF-A165 promoted proliferation from 72 to $96 \mathrm{~h}$ (Figure 2D and $\mathrm{E}, p<0.01$ ), migration (Figure $2 \mathrm{~F}$ and $\mathrm{G}$, $p=0.004$ ) and invasion (Figure $2 \mathrm{H}$ and $\mathrm{I}, p=0.002$ ) in pCMV-Dicer cells. The fact that VEGF-A165 counteracted the growth inhibition induced by Dicer in HCC cells implied that Dicer may inhibit HCC cell growth via the VEGF pathway.

\section{Bevacizumab Enhanced Dicer-Induced HCC Cell Growth Inhibition in vitro}

As Dicer inhibited HCC cell growth via the VEGF pathway, we investigated the effect of a blocking antiVEGF antibody (bevacizumab) on Dicer-induced HCC cell growth inhibition. As shown in Figure 3A, after incubation with $20 \mu \mathrm{g} / \mathrm{mL}$ bevacizumab, proliferation of pCMV-Dicer-infected SMMC-7721 cells significantly decreased from 48 to $72 \mathrm{~h}$ (Figure $3 \mathrm{~A}, p<0.01$ ), hence we used this concentration for subsequent experiment. Bevacizumab inhibited cell proliferation from 48 to $96 \mathrm{~h}$ (Figure $3 \mathrm{~B}$ and $\mathrm{C}, p<0.01$ ), migration (Figure $3 \mathrm{D}$ and $\mathrm{E}$, $p=0.000$ ) and invasion (Figure $3 \mathrm{~F}$ and $\mathrm{G}, p=0.000$ ) of pCMV-NC infected SMMC-7721 cells. Bevacizumab resulted in additive inhibition of cell proliferation from 24 to $96 \mathrm{~h}$ (Figure 3B and $\mathrm{C}, p<0.01$ ), migration (Figure $3 \mathrm{D}$ and $\mathrm{E}, p=0.004$ ) and invasion (Figure $3 \mathrm{~F}$ and $\mathrm{G}, p=0.018$ ) in pCMV-Dicer cells. Furthermore, the ability of pCMV-Dicer plus Bevacizumab HCC cells to proliferation from 48 to $96 \mathrm{~h}$ (Figure $3 \mathrm{~B}$ and $\mathrm{C}, p<0.01$ ), migration (Figure $3 \mathrm{D}$ and $\mathrm{E}, p=0.000$ ) and invasion (Figure $3 \mathrm{~F}$ and $\mathrm{G}, p=0.000$ ) obviously decreased compared to pCMV-NC HCC cells. Taken together, we conclude that bevacizumab suppressed proliferation, migration and invasion of HCC cells, thereby enhancing Dicer-induced HCC inhibition. 

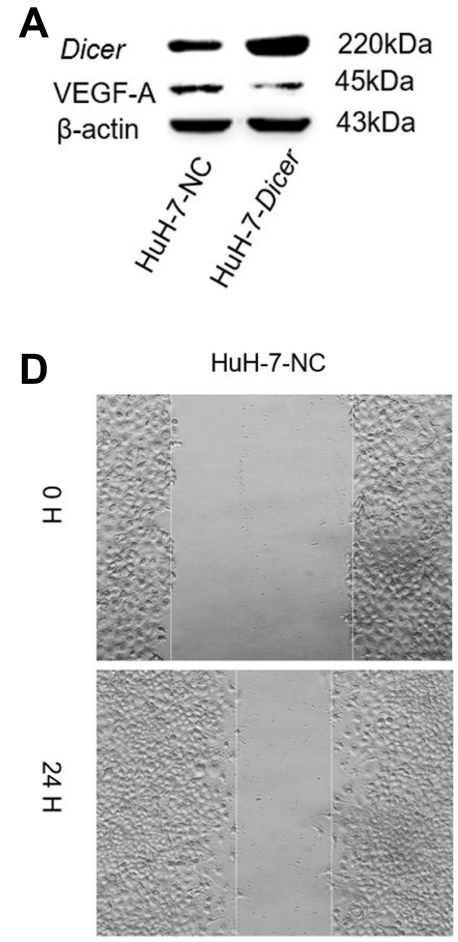

F

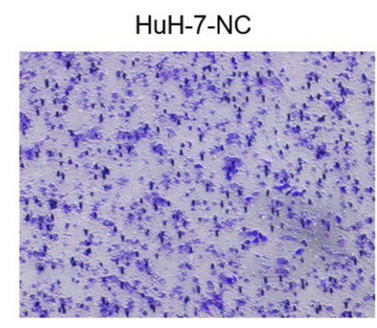

B

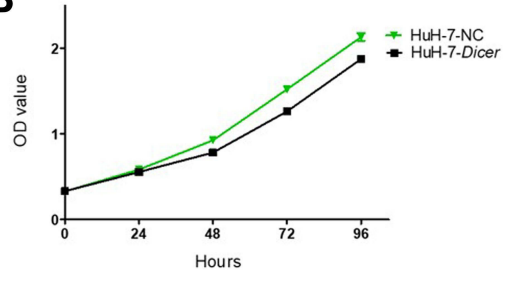

C

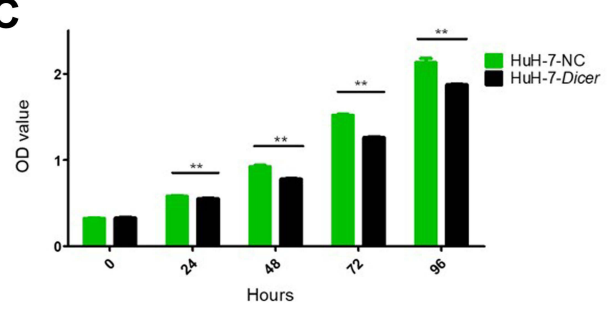

HuH-7-Dicer

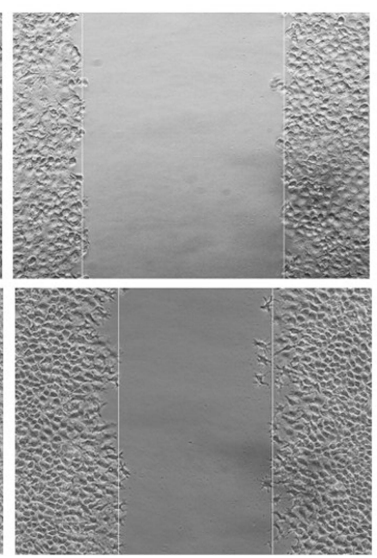

HuH-7-Dicer

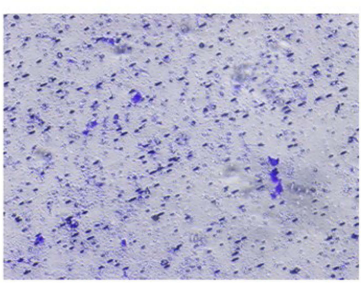

E

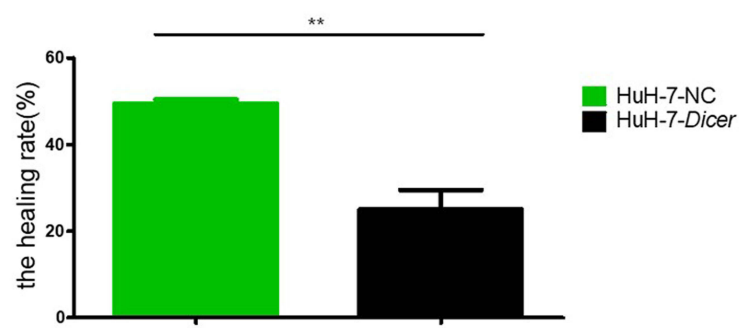

G

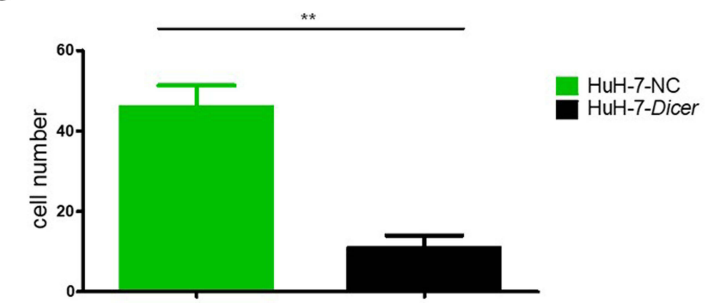

Figure I Dicer inhibits proliferation, migration and invasion of HuH-7 cells. (A) Western blot of Dicer and VEGF-A in HuH-7 cells; (B) The proliferation measurement by CCK-8 assay; (C) Quantification of results of (B); (D) The migration measurement by wound healing assay; (E) Quantification of results of (D); (F) The invasion measurement by transwell assay; (G) Quantification of results of $(\mathbf{F})$. ${ }^{* *} p \leq 0.01$.

Abbreviation: CCK-8, Cell Counting Kit-8.

\section{Bevacizumab Enhanced Dicer-Induced HCC Growth Inhibition in vivo}

In vivo, the mean tumor volume was significantly smaller in pCMV-Dicer xenografts than in pCMV-NC xenografts at 28 days after implantation (Figure $4 \mathrm{~A}-\mathrm{C}, p=0.001$ ). Throughout the course of treatment, the bevacizumab-related growth inhibition of pCMV-NC xenografts was achieved at 28 days, when compared to pCMV-NC xenografts (Figure $4 \mathrm{~A}-\mathrm{C}, p=0.005$ ). Moreover, the tumor volume of pCMV-Dicer xenografts treated with bevacizumab was significantly reduced from 14 to 28 days, when compared with that of pCMV-Dicer xenografts (Figure $4 \mathrm{~A}-\mathrm{C}, p<0.05$ ) and pCMV-NC xenografts (Figure $4 \mathrm{~A}-\mathrm{C}, p<0.05$ ). These data demonstrated that treatment with $20 \mathrm{mg} / \mathrm{kg}$ bevacizumab every three days enhanced Dicer-induced inhibition of HCC xenografts growth.
pCMV-Dicer xenografts showed lower VEGF-A expression compared to pCMV-NC xenografts, as determined with Western blotting (Figure 4D and $\mathrm{E}, p=0.001$ ). Bevacizumab decreased the level of VEGF-A in pCMVNC HCC xenografts (Figure 4D and E, $p=0.002$ ). Furthermore, the level of VEGF-A in pCMV-Dicer xenografts treated with bevacizumab was significantly than that in pCMV-NC xenografts (Figure 4D and E, $p=0.000$ ). Although VEGF-A expression in pCMV-Dicer xenografts treated with bevacizumab was reduced compared with that in pCMV-Dicer xenografts, the difference was not statistically significant (Figure 4D and E, $p=0.121$ ). CD31 expression was subsequently measured to compared the MVD difference upon bevacizumab treatment. As shown in Figure 4F and G, the MVD in pCMV-Dicer xenografts treated with bevacizumab was lower than that in pCMV- 
A

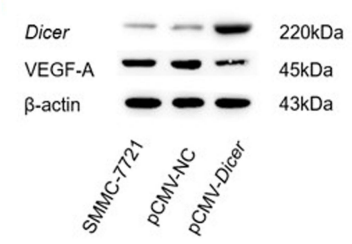

D

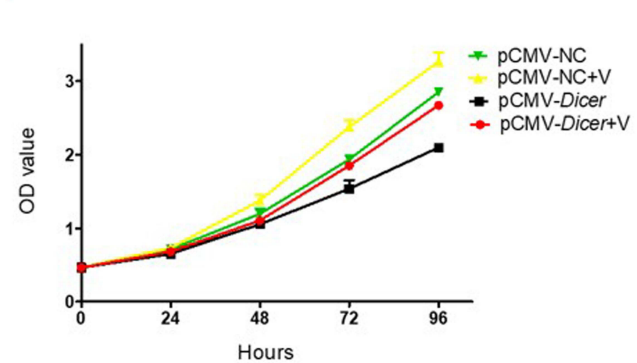

F

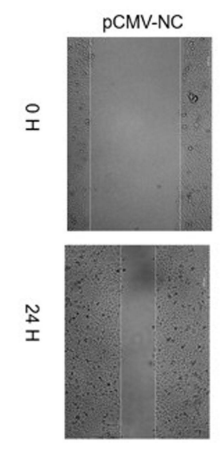

H

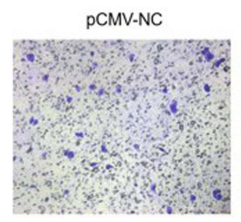

B

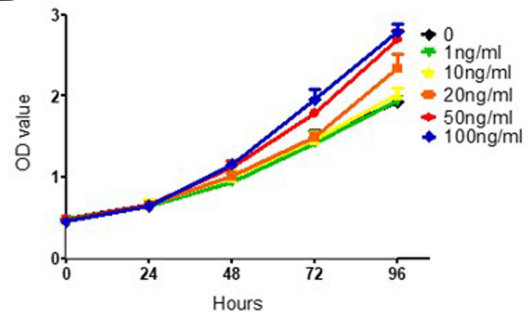

E
C

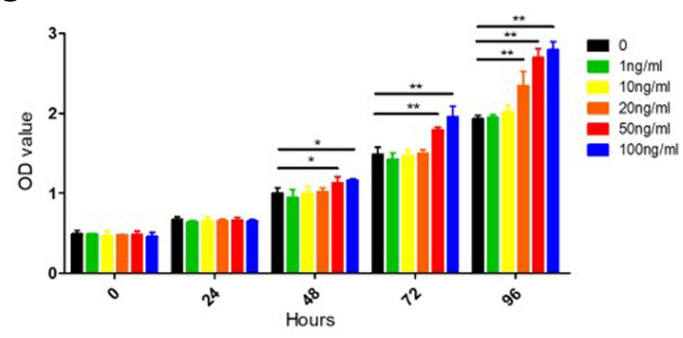

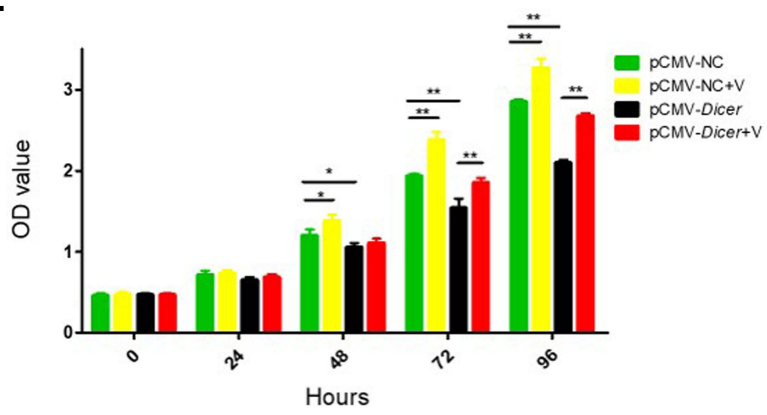

G

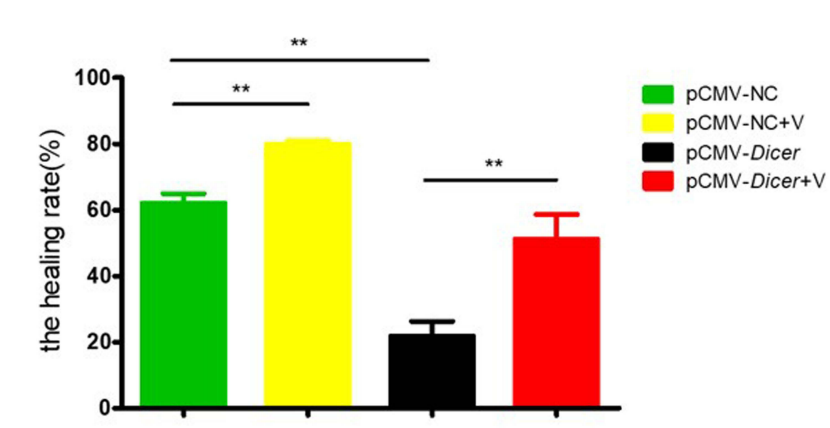

pCMV-Dicer+V
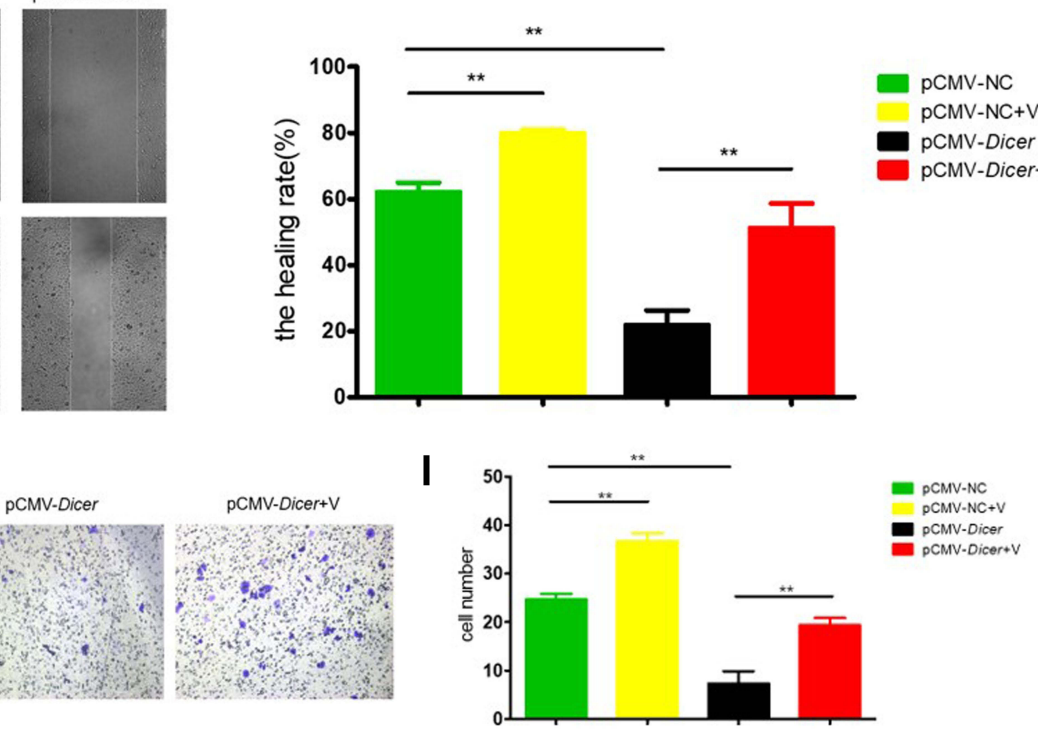

Figure 2 VEGF-AI 65 counteracts the inhibition induced by Dicer. (A) Western blot of Dicer and VEGF-A in SMMC-772I cells; (B) Determine the concentration of VEGFAI65 by CCK-8 assay; (C) Quantification of results of (B); (D) The proliferation measurement by CCK-8 assay; (E) Quantification of results of (D); (F) The migration

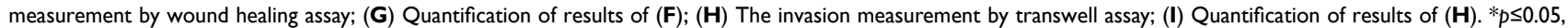
** $p \leq 0.01$.

Abbreviation: CCK-8, Cell Counting Kit-8.

Dicer xenografts (Figure $4 \mathrm{~F}$ and $\mathrm{G}, p=0.046$ ). In addition, tumor MVD was significantly decreased in pCMV-Dicer xenografts treated with bevacizumab compared with pCMV-NC xenografts (Figure $4 \mathrm{~F}$ and $\mathrm{G}, p=0.040$ ). Moreover, bevacizumab decreased the MVD in pCMVNC xenografts at a marginal statistical level (Figure $4 \mathrm{~F}$ and $\mathrm{G}, p=0.070$ ). These results indicated that bevacizumab and Dicer had a synergistic effect on suppression tumor angiogenesis.

\section{Discussion}

In the present study, we found that Dicer inhibited the growth of HCC cell in vitro and in vivo, as well as downregulated VEGF-A expression. VEGF-A165 counteracted the Dicer-induced HCC cell growth inhibition, while bevacizumab enhanced Dicer-induced HCC cell growth inhibition. Furthermore, bevacizumab and Dicer had a synergistic effect on the suppression tumor angiogenesis in HCC xenografts. Our data implied that Dicer could 

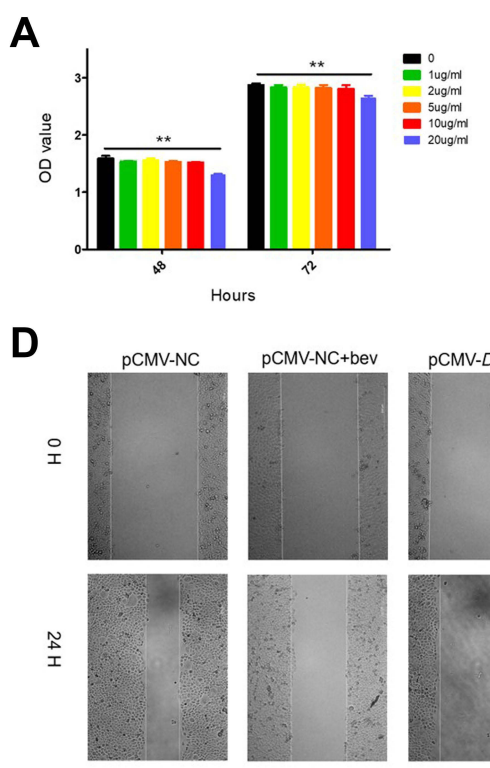

$\mathbf{F}$

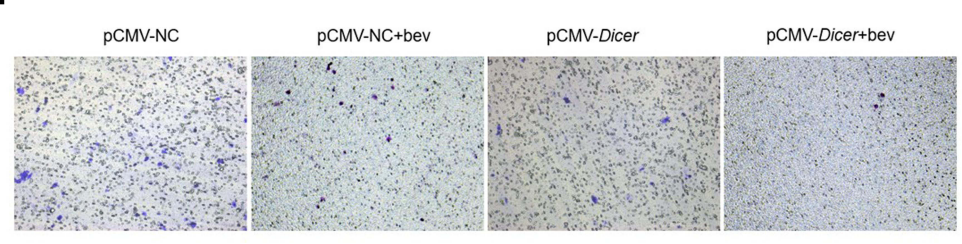

B

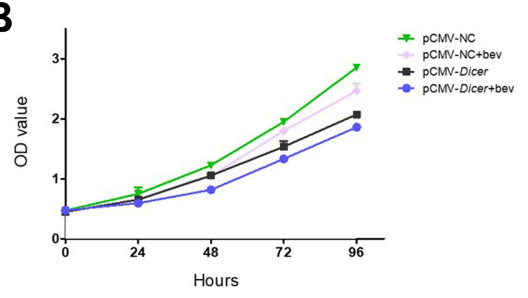

E
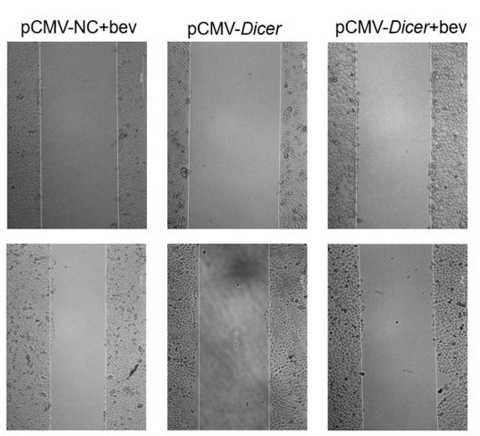
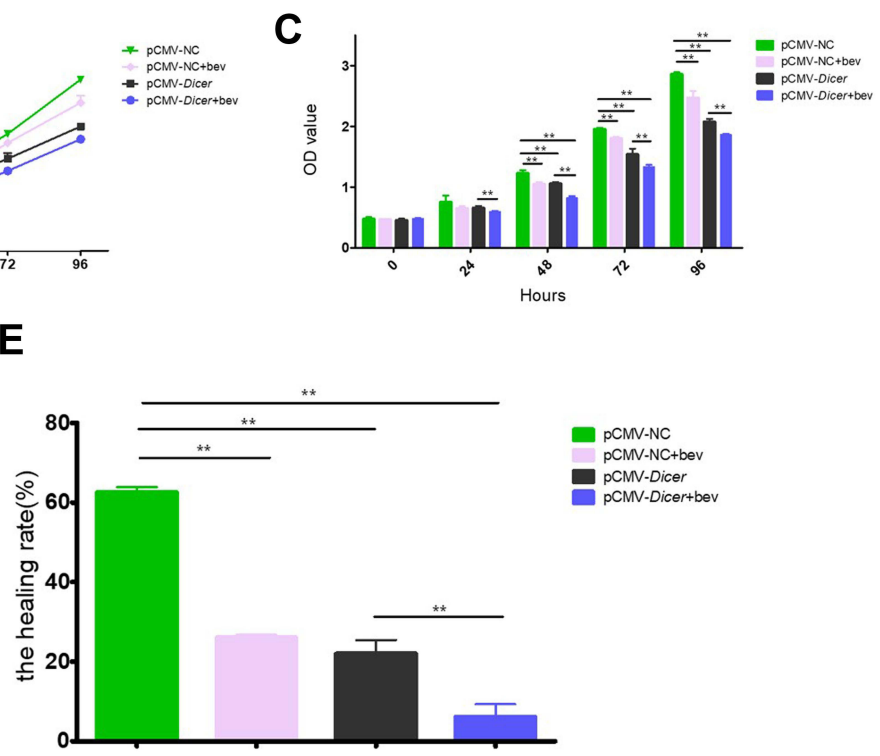

G

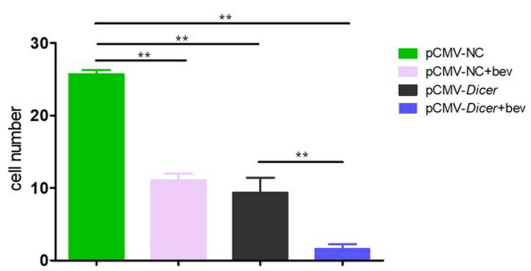

Figure 3 Bevacizumab enhances the inhibition induced by Dicer in vitro. (A) Determine the concentration of bevacizumab by CCK-8 assay; (B) The proliferation measurement by CCK-8 assay; (C) Quantification of results of (B); (D) The migration measurement by wound healing assay; (E) Quantification of results of (D); (F) The invasion measurement by transwell assay; (G) Quantification of results of $(\mathbf{F})$. ${ }^{* *} p \leq 0.01$.

Abbreviation: CCK-8, Cell Counting Kit-8.

enhance bevacizumab-induced HCC inhibition via the VEGF pathway.

HCC is a hypervascular tumor with a complex vascular network and several angiogenesis growth factors, VEGF, platelet-derived growth factor (PDGF) and basic fibroblast growth factor (bFGF), promote invasion and metastasis of HCC. ${ }^{5}$ VEGF contributes greatly to HCC pathogenesis, ${ }^{44,45}$ by promoting the growth of vascular endothelial cells (ECs) derived from arteries, veins and lymphatics. ${ }^{46}$ It prevents apoptosis via phosphatidylinositol (PI)-3 kinase-Akt pathway and promotes monocyte chemotaxis and colony formation of granulocyte-macrophage progenitor cells. ${ }^{47,48}$ It also induces an increase in vascular leakage mediated by calcium influx, ${ }^{49}$ thereby, potentially promoting tumor growth and metastasis. Bevacizumab, which is the most successful VEGF-neutralizing antibody, normalized tumor vessel growth leading to more efficient delivery of drugs in tumor microenvironment. When combined with chemotherapy, bevacizumab prolonged the survival of patients with lung, colon and breast cancer. ${ }^{16,50}$ In addition to chemotherapy synergism, bevacizumab significantly enhanced the outcome of treatment with the PD-L1 inhibitor atezolizumab in HCC patients by decreasing the activity of myeloid-derived suppressor cells and regulatory $\mathrm{T}$ cells as well as increasing cytotoxic T lymphocyte infiltration. ${ }^{19,51}$

Dicer, a key enzyme in the process of miRNA maturation, ${ }^{24-29}$ has been reported as an inconsistent prognostics factor for several cancers. We found that Dicer suppressed HCC growth by deregulating VEGF-A expression, consistent with a previous report showing that Dicer inhibited migration, invasion of clear cell RCC through suppressing VEGF-A expression. ${ }^{30}$ Because Dicer regulates miRNA expression, we performed miRNA microarray analysis to identify potential miRNAs that could affect VEGF-A expression. A total of 42 miRNAs were identified with a fold change $\geq 1.5$ upon Dicer overexpression (Supplementary Figure S2, Supplementary Table S1). Among the aberrantly expressed miRNAs, two miRNAs that suppress VEGF-A expression (miR-622, miR-378a$5 p$ ) were upregulated and three miRNAs that promote VEGF-A expression (miR-210-3p, miR-132-5p, miR874-3p) were downregulated, ${ }^{52-56}$ whereas one miRNA 
A
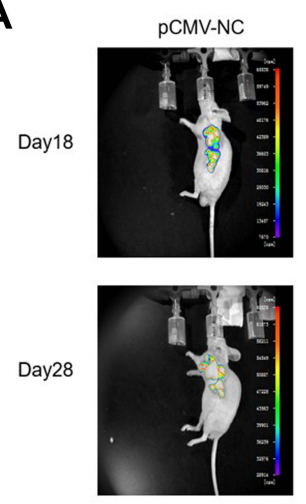

D

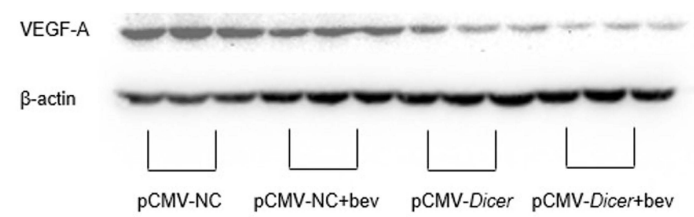

pCMV-NC+bev
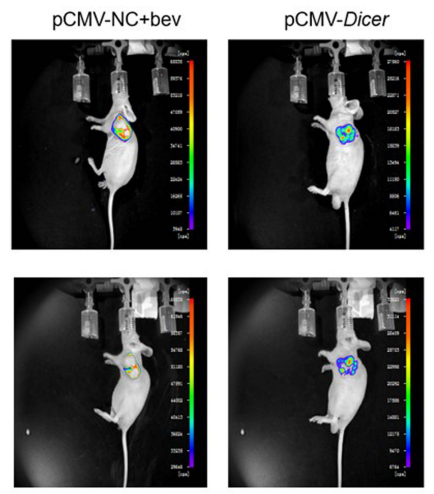

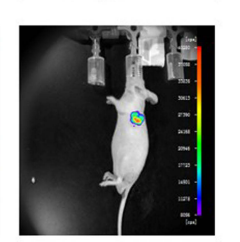

E

B

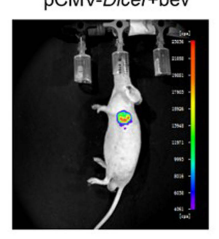

$45 \mathrm{kDa}$

C
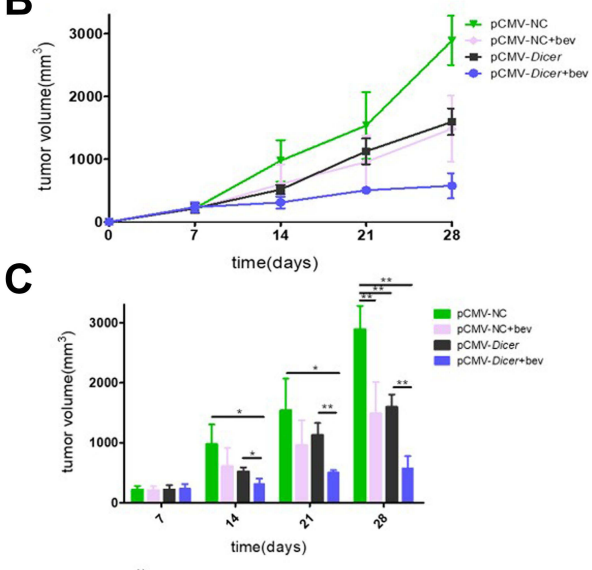

$43 \mathrm{kDa}$

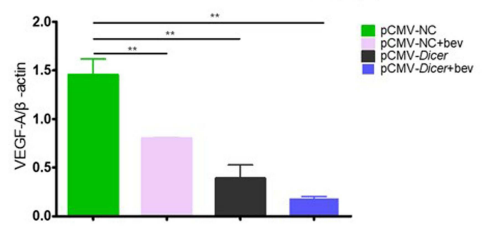

G

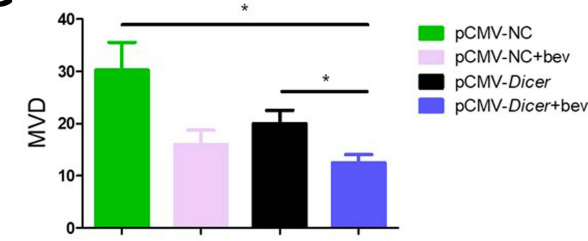

Figure 4 Bevacizumab enhances the inhibition induced by Dicer in vivo. (A) Fluorescence image of mice bearing HCC cells xenografts; (B) Tumor growth was monitored by measuring tumor volume for HCC xenografts; (C) Quantification of results of (B); (D) Western blot of VEGF-A in xenograft tumor tissue; (E) Quantification of results of (D). (F) Immunohistochemical analysis of CD3I; (G) Quantification of results of (F). $n=4$. ${ }^{*} p \leq 0.05,{ }^{* *} p \leq 0.01$.

that represses VEGF-A expression (miR-342-5p) was downregulated. $^{57,58}$ In summary, Dicer regulated five miRNAs that suppress VEGF-A expression and one miRNA that promotes VEGF-A expression. Obviously, the effect of suppression VEGF-A expression caused by Dicer was greater than that of promotion. In addition, four anti-angiogenic miRNAs related to other VEGF family members (miR-4485, miR-148a-5p, miR-338-3p, miR$374 b-5 p)$ were upregulated and one pro-angiogenic miRNA (miR-1247-5p) was downregulated. ${ }^{59-63}$ Therefore, Dicer may mediate angiogenesis through regulating these miRNAs related to VEGF.

Because our sample size of HCC xenografts was small, bevacizumab seemed to have the tendency to decrease VEGF-A level in pCMV-Dicer xenografts and MVD in pCMV-NC xenografts, albeit the difference was not statistically significant. In addition, the volume of pCMV-Dicer xenografts treated with bevacizumab significant decreased when compared with that of pCMV-NC xenografts at an early stage after implantation. These data indicate the clinical potential of Dicer to synergize with bevacizumab on HCC treatment. Several preclinical studies did suggest that the use of bevacizumab in combination with other agents may be a choice for HCC treatment. ${ }^{64}$ We found that calcitriol inhibited the growth of gastric cancer cells by inducing Dicer expression, ${ }^{65}$ however a suitable concentration of calcitriol should be assessed and validated preclinically in HCC xenografts.

\section{Conclusion}

Dicer enhanced bevacizumab-related inhibition of HCC cell and xenograft via the VEGF pathway, therefore factors that induce Dicer expression may be considered in combination with bevacizumab as an alternative option for HCC therapy.

\section{Abbreviations}

VEGF, vascular endothelial growth factor; HCC, hepatocellular carcinoma; MVD, microvessel density; PLC, primary liver cancer; HBV, hepatitis B virus; VEGFR, VEGF 
receptor; TKIs, tyrosine kinase inhibitors; $\mathrm{NCCN}$, National Comprehensive Cancer Network; mPFS, median progression-free survival; PD-L1, programmed cell death 1 ligand 1; miRNAs, microRNAs; 3'UTR, 3'-untranslated region; $\mathrm{RCC}$, renal cell carcinoma, FBS, fetal bovine serum; GFP, green fluorescent protein; CCK-8, cell counting kit-8; SPSS, Statistical Package for the Social Sciences; ANOVA, one-way analysis of variance; PDGF, platelet-derived growth factor; bFGF, basic fibroblast growth factor; ECs, vascular endothelial cells; PI, phosphatidylinositol.

\section{Data Sharing Statement}

The datasets used and/or analyzed during the current study are available from Zhanjun Guo upon reasonable request.

\section{Funding}

This work was supported by The Natural Science Foundation of Hebei Province of China (H2019206428) and the Foundation of Hebei Provincial Department of Science and Technology \& Hebei Medical University, Shijiazhuang, Hebei (2020TXZH03).

\section{Disclosure}

The authors report no competing interest in this work.

\section{References}

1. Sung H, Ferlay J, Siegel RL, et al. Global cancer statistics 2020: GLOBOCAN estimates of incidence and mortality worldwide for 36 cancers in 185 countries. CA Cancer J Clin. 2021;71(3):209-249. doi: $10.3322 /$ caac. 21660

2. Feng RM, Zong YN, Cao SM, Xu RH. Current cancer situation in China: good or bad news from the 2018 Global Cancer Statistics? Cancer Commun. 2019;39(1):22. doi:10.1186/s40880-019-0368-6

3. Chen Y, Tian Z. HBV-induced immune imbalance in the development of HCC. Front Immunol. 2019;10:2048. doi:10.3389/ fimmu.2019.02048

4. Zhang FP, Huang YP, Luo WX, et al. Construction of a risk score prognosis model based on hepatocellular carcinoma microenvironment. World J Gastroenterol. 2020;26(2):134-153. doi:10.3748/wjg. v26.i2.134

5. Gong X, Qin S. Study progression of anti-angiogenetic therapy and its combination with other agents for the treatment of advanced hepatocellular carcinoma. Hepatobiliary Surg Nutr. 2018;7(6):466-474. doi:10.21037/hbsn.2018.11.04

6. Johnson PJ, Qin S, Park JW, et al. Brivanib versus sorafenib as firstline therapy in patients with unresectable, advanced hepatocellular carcinoma: results from the randomized Phase III BRISK-FL study. $J$ Clin Oncol. 2013;31(28):3517-3524. doi:10.1200/JCO.2012.48.4410

7. Llovet JM, Decaens T, Raoul JL, et al. Brivanib in patients with advanced hepatocellular carcinoma who were intolerant to sorafenib or for whom sorafenib failed: results from the randomized phase III BRISK-PS study. J Clin Oncol. 2013;31(28):3509-3516. doi:10.1200/ JCO.2012.47.3009
8. Cheng AL, Kang YK, Lin DY, et al. Sunitinib versus sorafenib in advanced hepatocellular cancer: results of a randomized phase III trial. J Clin Oncol. 2013;31(32):4067-4075. doi:10.1200/ JCO.2012.45.8372

9. Cainap C, Qin S, Huang WT, et al. Linifanib versus Sorafenib in patients with advanced hepatocellular carcinoma: results of a randomized phase III trial. J Clin Oncol. 2015;33(2):172-179. doi:10.1200/ JCO.2013.54.3298

10. Zhu AX, Kudo M, Assenat E, et al. Effect of everolimus on survival in advanced hepatocellular carcinoma after failure of sorafenib: the EVOLVE-1 randomized clinical trial. JAMA. 2014;312(1):57-67. doi:10.1001/jama.2014.7189

11. Kang YK, Yau T, Park JW, et al. Randomized phase II study of axitinib versus placebo plus best supportive care in second-line treatment of advanced hepatocellular carcinoma. Ann Oncol. 2015;26(12):2457-2463. doi:10.1093/annonc/mdv388

12. Cheng AL, Kang YK, Chen Z, et al. Efficacy and safety of sorafenib in patients in the Asia-Pacific region with advanced hepatocellular carcinoma: a phase III randomised, double-blind, placebo-controlled trial. Lancet Oncol. 2009;10(1):25-34. doi:10.1016/S1470-2045(08) 70285-7

13. Kudo M, Finn RS, Qin S, et al. Lenvatinib versus sorafenib in firstline treatment of patients with unresectable hepatocellular carcinoma: a randomised phase 3 non-inferiority trial. Lancet. 2018;391 (10126):1163-1173. doi:10.1016/S0140-6736(18)30207-1

14. Zhang XH, Cao MQ, Li XX, Zhang T. Apatinib as an alternative therapy for advanced hepatocellular carcinoma. World $J$ Hepatol. 2020;12(10):766-774. doi:10.4254/wjh.v12.i10.766

15. Zhu AX, Kang YK, Yen CJ, et al. Ramucirumab after sorafenib in patients with advanced hepatocellular carcinoma and increased alphafetoprotein concentrations (REACH-2): a randomised, double-blind, placebo-controlled, phase 3 trial. Lancet Oncol. 2019;20(2):282-296. doi:10.1016/S1470-2045(18)30937-9

16. Presta LG, Chen H, O'Connor SJ, et al. Humanization of an antivascular endothelial growth factor monoclonal antibody for the therapy of solid tumors and other disorders. Cancer Res. 1997;57 (20):4593-4599.

17. Siegel AB, Cohen EI, Ocean A, et al. Phase II trial evaluating the clinical and biologic effects of bevacizumab in unresectable hepatocellular carcinoma. $J$ Clin Oncol. 2008;26(18):2992-2998. doi:10.1200/JCO.2007.15.9947

18. Rizvi S, Wang J, El-Khoueiry AB. Liver cancer immunity. Hepatology. 2021;73(Suppl 1):86-103. doi:10.1002/hep.31416

19. Yang $X$, Wang D, Lin J, Yang $X$, Zhao H. Atezolizumab plus bevacizumab for unresectable hepatocellular carcinoma. Lancet Oncol. 2020;21(9):e412. doi:10.1016/S1470-2045(20)30430-7

20. Bartel DP. MicroRNAs: target recognition and regulatory functions. Cell. 2009;136(2):215-233. doi:10.1016/j.cell.2009.01.002

21. Lee RC, Feinbaum RL, Ambros V. The C. elegans heterochronic gene lin-4 encodes small RNAs with antisense complementarity to lin-14. Cell. 1993;75(5):843-854. doi:10.1016/0092-8674(93)90529-Y

22. Bagga S, Bracht J, Hunter S, et al. Regulation by let-7 and lin-4 miRNAs results in target mRNA degradation. Cell. 2005;122(4):553563. doi:10.1016/j.cell.2005.07.031

23. Kim VN. MicroRNA biogenesis: coordinated cropping and dicing. Nat Rev Mol Cell Biol. 2005;6(5):376-385. doi:10.1038/nrm1644

24. Zhang LI, Wang C, Liu S, Zhao Y, Liu C, Guo Z. Prognostic significance of Dicer expression in hepatocellular carcinoma. Oncol Lett. 2016;11(6):3961-3966. doi:10.3892/ol.2016.4547

25. Ma X, Fan Y, Gao Y, et al. Dicer is down-regulated in clear cell renal cell carcinoma and in vitro Dicer knockdown enhances malignant phenotype transformation. Urol Oncol. 2014;32(1):46-e9. doi:10.1016/j.urolonc.2013.06.011

26. Zhang J, Zhang XH, Wang CX, et al. Dysregulation of microRNA biosynthesis enzyme Dicer plays an important role in gastric cancer progression. Int J Clin Exp Pathol. 2014;7(4):1702-1707. 
27. Dedes KJ, Natrajan R, Lambros MB, et al. Down-regulation of the miRNA master regulators Drosha and Dicer is associated with specific subgroups of breast cancer. Eur J Cancer. 2011;47(1):138-150. doi:10.1016/j.ejca.2010.08.007

28. Faggad A, Kasajima A, Weichert W, et al. Down-regulation of the microRNA processing enzyme Dicer is a prognostic factor in human colorectal cancer. Histopathology. 2012;61(4):552-561. doi:10.1111/ j.1365-2559.2011.04110.x

29. Zhu DX, Fan L, Lu RN, et al. Downregulated Dicer expression predicts poor prognosis in chronic lymphocytic leukemia. Cancer Sci. 2012;103(5):875-881. doi:10.1111/j.1349-7006.2012.02234.x

30. Chen YS, Meng F, Li HL, et al. Dicer suppresses MMP-2-mediated invasion and VEGFA-induced angiogenesis and serves as a promising prognostic biomarker in human clear cell renal cell carcinoma. Oncotarget. 2016;7(51):84299-84313. doi:10.18632/oncotarget.12520

31. Smith HO, Danner DB, Deich RA. Genetic transformation. Annu Rev Biochem. 1981;50:41-68. doi:10.1146/annurev.bi.50.070181.000353

32. Towbin H, Staehelin T, Gordon J. Electrophoretic transfer of proteins from polyacrylamide gels to nitrocellulose sheets: procedure and some applications. Proc Natl Acad Sci U S A. 1979;76(9):43504354. doi:10.1073/pnas.76.9.4350

33. Ouyang Y, Li Y, Huang Y, et al. CircRNA circPDSS1 promotes the gastric cancer progression by sponging miR-186-5p and modulating NEK2. J Cell Physiol. 2019;234(7):10458-10469. doi:10.1002/ jcp. 27714

34. Nanashima N, Horie K, Yamada T, Shimizu T, Tsuchida S. Hair keratin KRT81 is expressed in normal and breast cancer cells and contributes to their invasiveness. Oncol Rep. 2017;37(5):2964-2970. doi:10.3892/or.2017.5564

35. Meng F, Wang F, Wang L, Wong SC, Cho WC, Chan LW. MiR-30a-5p overexpression may overcome EGFR-inhibitor resistance through regulating PI3K/AKT signaling pathway in non-small cell lung cancer cell lines. Front Genet. 2016;7:197. doi:10.3389/fgene.2016.00197

36. Shi X, Liu Q, Liu H, Deng D, Qiao F, Wu Y. Effects of shRNA targeting maspin on the invasion of extravillous trophoblast cell. Am $J$ Perinatol. 2017;34(10):966-973. doi:10.1055/s-0037-1601458

37. National Research Council (U.S.). Committee for the Update of the Guide for the Care and Use of Laboratory Animals., Institute for Laboratory Animal Research (U.S.), National Academies Press (U.S.). Guide for the Care and Use of Laboratory Animals. 8th ed. Washington, D.C.: National Academies Press; 2011. Available from: http://www. ncbi.nlm.nih.gov/books/NBK54050. Accessed December 24, 2021.

38. Shirakami Y, Shimizu M, Adachi S, et al. (-)-Epigallocatechin gallate suppresses the growth of human hepatocellular carcinoma cells by inhibiting activation of the vascular endothelial growth factor-vascular endothelial growth factor receptor axis. Cancer Sci. 2009;100 (10):1957-1962. doi:10.1111/j.1349-7006.2009.01241.x

39. Chen Z, Qian X, Chen S, Fu X, Ma G, Zhang A. Akkermansia muciniphila enhances the antitumor effect of cisplatin in Lewis lung cancer mice. J Immunol Res. 2020;2020:2969287. doi:10.1155/ 2020/2969287

40. Weidner N. Current pathologic methods for measuring intratumoral microvessel density within breast carcinoma and other solid tumors. Breast Cancer Res Treat. 1995;36(2):169-180. doi:10.1007/ BF00666038

41. Nassar FJ, Talhouk R, Zgheib NK, et al. microRNA expression in ethnic specific early stage breast cancer: an integration and comparative analysis. Sci Rep. 2017;7(1):16829. doi:10.1038/s41598-01716978-y

42. Matsumoto T, Claesson-Welsh L. VEGF receptor signal transduction. Sci STKE. 2001;2001(112):re21. doi:10.1126/stke.2001.112.re21

43. Sheen IS, Jeng KS, Shih SC, et al. Clinical significance of the expression of isoform 165 vascular endothelial growth factor mRNA in noncancerous liver remnants of patients with hepatocellular carcinoma. World J Gastroenterol. 2005;11(2):187-192. doi:10.3748/wjg.v11.i2.187
44. Carmeliet P, Jain RK. Molecular mechanisms and clinical applications of angiogenesis. Nature. 2011;473(7347):298-307. doi:10.1038/ nature 10144

45. Scartozzi M, Faloppi L, Svegliati Baroni G, et al. VEGF and VEGFR genotyping in the prediction of clinical outcome for $\mathrm{HCC}$ patients receiving sorafenib: the ALICE-1 study. Int J Cancer. 2014;135 (5):1247-1256. doi:10.1002/ijc. 28772

46. Ferrara N, Davis-Smyth T. The biology of vascular endothelial growth factor. Endocr Rev. 1997;18(1):4-25. doi:10.1210/ edrv.18.1.0287

47. Gerber HP, McMurtrey A, Kowalski J, et al. Vascular endothelial growth factor regulates endothelial cell survival through the phosphatidylinositol 3'-kinase/Akt signal transduction pathway. Requirement for Flk-1/KDR activation. J Biol Chem. 1998;273 (46):30336-30343. doi:10.1074/jbc.273.46.30336

48. Clauss M, Gerlach M, Gerlach H, et al. Vascular permeability factor: a tumor-derived polypeptide that induces endothelial cell and monocyte procoagulant activity, and promotes monocyte migration. $J$ Exp Med. 1990;172(6):1535-1545. doi:10.1084/jem.172.6.1535

49. Bates DO, Curry FE. Vascular endothelial growth factor increases microvascular permeability via a $\mathrm{Ca}(2+)$-dependent pathway. $\mathrm{Am} \mathrm{J}$ Physiol. 1997;273(2 Pt 2):H687-H694. doi:10.1152/ ajpheart.1997.273.2.H687

50. Jain RK, Duda DG, Clark JW, Loeffler JS. Lessons from phase III clinical trials on anti-VEGF therapy for cancer. Nat Clin Pract Oncol. 2006;3(1):24-40. doi:10.1038/ncponc0403

51. Yang J, Yan J, Liu B. Targeting VEGF/VEGFR to Modulate Antitumor Immunity. Front Immunol. 2018;9:978. doi:10.3389/ fimmu.2018.00978

52. Fang Y, Sun B, Wang J, Wang Y. miR-622 inhibits angiogenesis by suppressing the CXCR4-VEGFA axis in colorectal cancer. Gene. 2019;699:37-42. doi:10.1016/j.gene.2019.03.004

53. Cui Z, Liu QL, Sun SQ, et al. MiR-378a-5p inhibits angiogenesis of oral squamous cell carcinoma by targeting KLK4. Neoplasma. 2020;67(1):85-92. doi:10.4149/neo_2019_190306N191

54. Popov TM, Giragosyan S, Petkova V, et al. Proangiogenic signature in advanced laryngeal carcinoma after microRNA expression profiling. Mol Biol Rep. 2020;47(7):5651-5655. doi:10.1007/s11033-02005250-8

55. Ghaffari-Makhmalbaf $P$, Sayyad $M$, Pakravan $K$, et al. Docosahexaenoic acid reverses the promoting effects of breast tumor cell-derived exosomes on endothelial cell migration and angiogenesis. Life Sci. 2021;264:118719. doi:10.1016/j.1fs.2020.118719

56. Huang Y, Han Y, Guo R, et al. Long non-coding RNA FER1L4 promotes osteogenic differentiation of human periodontal ligament stromal cells via miR-874-3p and vascular endothelial growth factor A. Stem Cell Res Ther. 2020;11(1):5. doi:10.1186/s13287-019-1519-z

57. Yan XC, Cao J, Liang L, et al. miR-342-5p is a notch downstream molecule and regulates multiple angiogenic pathways including notch, vascular endothelial growth factor and transforming growth factor $\beta$ signaling. $J$ Am Heart Assoc. 2016;5:2. doi:10.1161/ JAHA.115.003042

58. Ray SL, Coulson DJ, Yeoh MLY, et al. The role of miR-342 in vascular health. study in subclinical cardiovascular disease in mononuclear cells, plasma, inflammatory cytokines and PANX2. Int J Mol Sci. 2020;21(19):7217. doi:10.3390/ijms21197217

59. Yang B, Jing C, Wang J, et al. Identification of microRNAs associated with lymphangiogenesis in human gastric cancer. Clin Transl Oncol. 2014;16(4):374-379. doi:10.1007/s12094-013-1081-6

60. Kasiviswanathan D, Chinnasamy Perumal R, Bhuvaneswari S, et al. Interactome of miRNAs and transcriptome of human umbilical cord endothelial cells exposed to short-term simulated microgravity. NPJ Microgravity. 2020;6:18. doi:10.1038/s41526-020-00108-6

61. Zhang T, Liu W, Zeng XC, et al. Down-regulation of microRNA-338$3 \mathrm{p}$ promoted angiogenesis in hepatocellular carcinoma. Biomed Pharmacother. 2016;84:583-591. doi:10.1016/j.biopha.2016.09.056 
62. Ji H, Zhang X. RPL38 regulates the proliferation and apoptosis of gastric cancer via miR-374b-5p/VEGF signal pathway. Onco Targets Ther. 2020;13:6131-6141. doi:10.2147/OTT.S252045

63. Ayaz L, Dinc E. Evaluation of microRNA responses in ARPE-19 cells against the oxidative stress. Cutan Ocul Toxicol. 2018;37 (2):121-126. doi:10.1080/15569527.2017.1355314

64. Kubota M, Shimizu M, Baba A, et al. Combination of bevacizumab and acyclic retinoid inhibits the growth of hepatocellular carcinoma xenografts. J Nutr Sci Vitaminol. 2014;60(5):357-362. doi:10.3177/ jnsv.60.357
65. Wu J, Zhao Q, Zhao Y, Zhang X, Tian Y, Guo Z. Dicer increases the indication for trastuzumab treatment in gastric cancer patients via overexpression of human epidermal growth factor receptor 2. Sci Rep. 2021;11(1):6993. doi:10.1038/s41598-021-86485-8

\section{Publish your work in this journal}

The Journal of Hepatocellular Carcinoma is an international, peerreviewed, open access journal that offers a platform for the dissemination and study of clinical, translational and basic research findings in this rapidly developing field. Development in areas including, but not limited to, epidemiology, vaccination, hepatitis therapy, pathology and molecular tumor classification and prognostication are all considered for publication. The manuscript management system is completely online and includes a very quick and fair peer-review system, which is all easy to use. Visit http://www.dovepress.com/ testimonials.php to read real quotes from published authors.

Submit your manuscript here: https://www.dovepress.com/journal-of-hepatocellular-carcinoma-journal 